\title{
VARIABILITY OF DRIVING PERFORMANCE DURING MICROSLEEPS
}

\author{
Amit Paul $^{1}$, Linda Ng Boyle ${ }^{1,3}$, Jon Tippin ${ }^{2}$, Matthew Rizzo ${ }^{1,2,3}$ \\ (1) College of Engineering and (2) Medicine (3) Public Policy Center \\ University of Iowa, Iowa City, Iowa, USA \\ E-mail: linda-boyle@uiowa.edu
}

\begin{abstract}
Summary: This study aimed to evaluate the value of measuring microsleeps as an indicator of driving performance impairment in drowsy drivers with sleep disorders. Drivers with sleep disorders such as obstructive sleep apnea/hypopena syndrome (OSAHS) are at increased risk for driving performance errors due to microsleep episodes, which presage sleep onset. To meet this aim, we tested the hypothesis that OSAHS drivers show impaired control over vehicle steering, lane position and velocity during microsleep episodes compared to when they are driving without microsleeps on similar road segments. A microsleep is defined as a 3-14 sec episode during which 4-7 Hz (theta) activity replaces the waking 8-13 $\mathrm{Hz}$ (alpha) background rhythm. Microsleep episodes were identified in the electroencephalography (EEG) record by a neurologist certified by the American Board of Sleep Medicine. Twenty-four drivers with OSAHS were tested using simulated driving scenarios. Steering variability, lane position variability, acceleration and velocity measures were assessed in the periods during a microsleep, immediately preceding (pre) microsleep, and immediately following (post) microsleep. In line with our introductory hypothesis, drivers with OSAHS did show significantly greater variation in steering and lane position during the microsleep episodes compared to the periods pre and post microsleep. The results indicate that identification of microsleep episodes can provide a marker for declining vehicle control of drivers with OSAHS.
\end{abstract}

\section{INTRODUCTION}

National Highway Transportation Safety Administration estimates indicate that sleepiness causes about 100,000 police-reported crashes, and $4 \%$ of all fatal vehicle crashes each year (NHTSA, 2005). Sleep disorders such as insomnia, narcolepsy and OSAHS increase the risk of sleeprelated crashes. Drivers with OSAHS - which is the cessation of airflow during sleep (American Thoracic Society, 1994) - are at particularly high risk of sleep-related crashes (Findley et al., 1995; George et al., 1987; Young et al., 1993). These drivers are more likely to have microsleep episodes, the lapses from wakefulness into sleep that last a few seconds and can be demonstrated with EEG recordings. Microsleeps are a marker for excessive daytime sleepiness and have been associated with poor simulated driving performance (Risser et al., 2000).

To develop countermeasures to sleep-related driving errors, it is necessary to understand driving performance profiles due to sleepiness. This study tested the hypothesis that drivers with OSAHS show worse driving performance during microsleep episodes compared to driving performance when no microsleep occurs. Microsleep bouts presage sleep-onset and last typically 3-14 seconds. Identification of microsleep episodes and associated variations in driving performance (e.g., steering, lane position, velocity) would increase our ability to develop alerting and warning devices to aid at-risk sleepy drivers. 


\section{METHODOLOGY}

\section{Participants}

Twenty-four legally licensed adult drivers (mean age $=47.9$ years, $\mathrm{SD}=9.71$, range $30-65$ years old) with medically diagnosed OSAHS. They were compensated $\$ 50$ for their time and effort.

\section{Experimental Procedure}

Participants drove a fixed-based, high fidelity driving simulator called SIREN (Simulator for Interdisciplinary Research in Ergonomics and Neuroscience) (Rizzo et al., 2000). The participants encountered a two-lane highway with interactive traffic and road segments that consisted of straight and curvy roads. The drive lasted approximately 60 minutes, with participants driving below the speed limit of $55 \mathrm{mph}$. The simulated drives were purposely uneventful (involving minimal traffic, few intersections, no lead vehicles, and no other external distractions) to induce drowsiness.

\section{Dependent Measures}

Lane Variability: Lane position variability is measured with the standard deviation of lane position (SDLP). SDLP is an index of road tracking error or "weaving." This measure will identify whether a driver's ability to control the vehicle is maintained or diminished. This measure was calculated over the intervals of interest (microsleep periods).

Steering Variability: Variability association with steering was measured as the standard deviation of steering wheel angle (SDSTW). This variable has been widely used as an index of steering performance (Liu et al., 1999; Mollenhauer et al., 2004).

Time to lane crossing (TLC). TLC is the time required for a vehicle to run off the road boundary (road shoulder or oncoming traffic lane) assuming the current steering wheel angle is held constant and there is no further steering intervention by the driver (Lin \& Ulsoy, 1995). An approximation method (i.e., assuming constant lateral velocity) was used as follows:

$$
T L C_{\text {lateral }}=\min \left\{\frac{D_{l}}{\dot{D}_{l}}, \frac{D_{r}}{\dot{D}_{r}}\right\}
$$

Where $D_{l}$ and $D_{r}$ are the lateral distance between the front wheel and the lane boundary (from left and right), and $\dot{D}_{l}$ and $\dot{D}_{r}$ are the lateral velocities towards left or right.

\section{Independent Measures}

Microsleep periods. A microsleep is defined as a 3-14 sec episode during which 4-7 Hz (theta) activity replaced the waking 8-13 Hz (alpha) background rhythm. Microsleep events were interpreted from EEG recordings measured from a Neurofax EEG-2100 (Nihon Khoden Corp, Tokyo) by an American Board of Sleep Medicine-accredited neurologist. For this study, the dependent measures were analyzed across three intervals: 
- Pre: Three-second interval immediately preceding a microsleep

- During: Period of the microsleep interval

- Post: Three-second interval immediately following a microsleep

The three-second duration pre- and post-microsleep intervals were used (1) to minimize the influence of driving factors other than microsleeps; and (2) to account for the number of shorter microsleep durations. More microsleep events of shorter duration were expected, because duration follows a Poisson distribution (see Figure 1); the mode of this Poisson distribution is around three seconds and thus this interval is used.

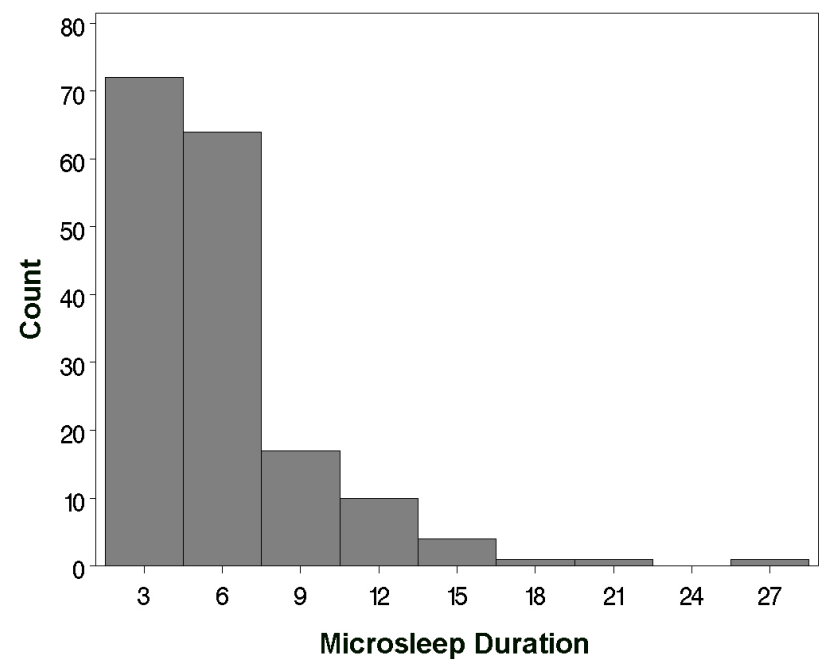

Figure 1. Distribution of microsleep durations

Road segments. Two road segments types were analyzed: straight roads and curved roads. The curved road segments consisted of three left and three right turns, each with a 600-meter radii.

\section{Experimental Design}

We used a case-crossover design to assess the changes in behavior associated with microsleeps (Maclure, 1991). The case-crossover method allows assessment of the effect of microsleeps during the period when they are present (the case) as opposed to absent (the crossover control) during the drive (Marshall, 1993). To evaluate driving performance during microsleeps, we established control (non-microsleep) episodes during the drive that were matched to the case (microsleep) episodes. A 2:1 matched approach was used to compare the time periods during, pre and post microsleep (as shown in Figure 2).

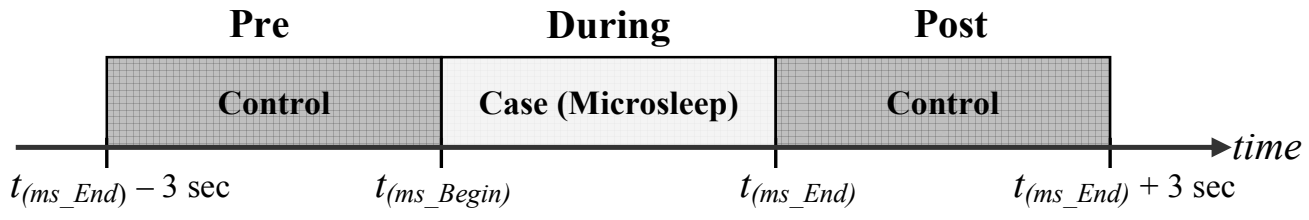

Figure 2. Case-crossover design - match based on time before and after the microsleep episode 
A repeated measures Analysis of Variance (ANOVAs) was used to investigate the differences across microsleep periods. Intercept and the number of microsleeps were included as random effects. Given that the dependent measures needed to follow a normal distribution for analysis, the following transformations were performed:

$\begin{array}{ll}\text { Lane Position SD: } & \sqrt[4]{S D L P} \\ \text { Steering Standard Deviation: } & \ln (1+S D S T W) \\ \text { Minimum Time to Lane Crossing }(T L C): & \sqrt{\text { Minimum TLC }}\end{array}$

A nonparametric test (such as Kruskal-Wallis) was not used because it assumes that the response variable does not follow any distribution nor can the variables be transformed in any way. However, dependent variables in these studies were easily transformed to a normal form.

\section{DATA ANALYSIS AND RESULTS}

Steering Variability. Average steering standard deviation during microsleeps was 2.1 radians, and 1.5 radians post microsleep regardless of road segment type. There were no differences in steering variability associated with road segments $(\mathrm{F}(1,159)=3.88, \mathrm{p}=0.051)$. Steering variability was significantly different among the three periods (pre, during and post microsleep) $(\mathrm{F}(2,336)=17.07, \mathrm{p}<0.0001)$, however, with variability being higher during microsleeps when compared to the pre- and post-microsleep episodes. In summary, drivers with OSAHS showed greater steering variation during microsleep periods, regardless of the road type (as shown in Figure 3).

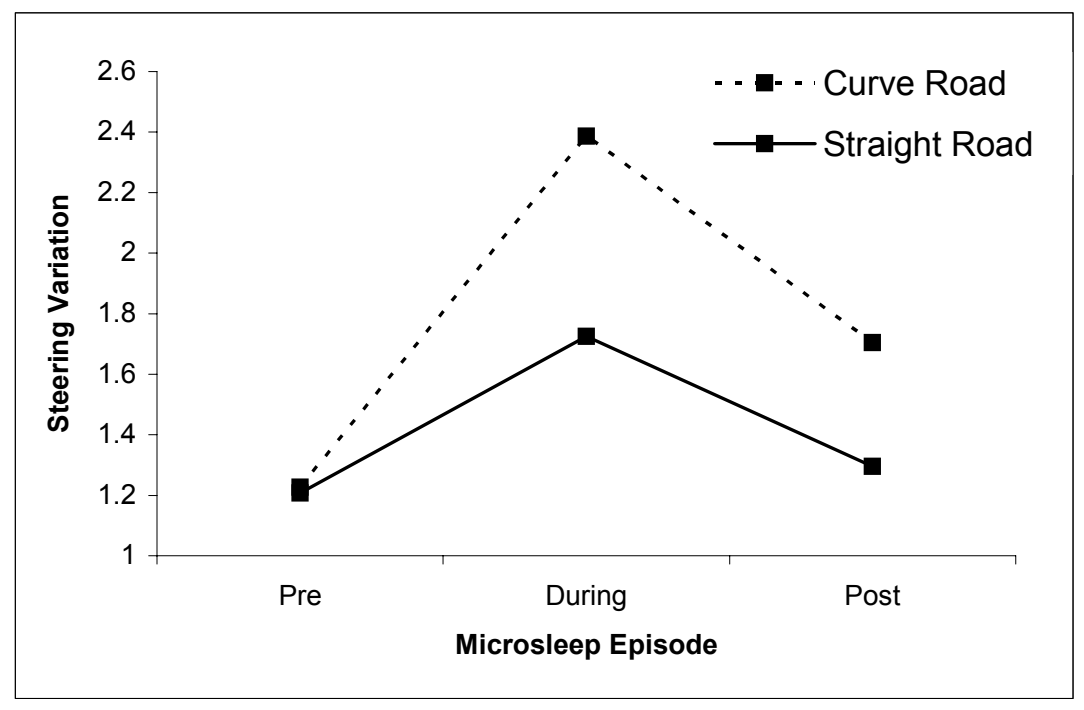

Figure 3. The effect microsleep on steering variation for straight and curved roads

Lane Position Variability. Lane position variation was also different among the microsleep and the pre- and post-microsleep intervals $(\mathrm{F}(2,336)=29.76, \mathrm{p}<0.0001)$. The lane position variability was greater during the microsleeps compared to the pre- and post-microsleep intervals (as shown in Figure 4). OSAHS drivers had a lane position variation of 0.16 during microsleep episodes and of 0.09 in the period after a microsleep. Lane position variation was significantly 
higher on curved road segments compared to straight roads $(\mathrm{F}(1,158)=4.79, \mathrm{p}<0.05)$. However, there were no interactions $(\mathrm{F}(2,336=0.69, \mathrm{p}>0.05)$ with road segment types.

Minimum Time to Lane Crossing (TLC). The minimum TLC is an indicator of unsafe driving and was assessed in the pre-, during and post-microsleep intervals. The main effect of minimum TLC was significantly different among the periods of pre-, during and post-microsleep $(\mathrm{F}(2,336)=$ 8.73, $\mathrm{p}<0.0005$ ). On average, minimum $T L C$ for OSAHS drivers was 1.7 seconds during microsleep and 2.6 seconds post-microsleep (see Figure 5). Minimum TLC was significantly lower during the microsleeps compared to pre- and post-microsleep periods. Minimum TLC was significantly lower on curved roads compared to straight roads $(\mathrm{F}(1,159)=68.34, \mathrm{p}<0.0001)$. However, there were no interactions $(\mathrm{F}(2,336=2.36, \mathrm{p}>0.05)$ with road segment types.

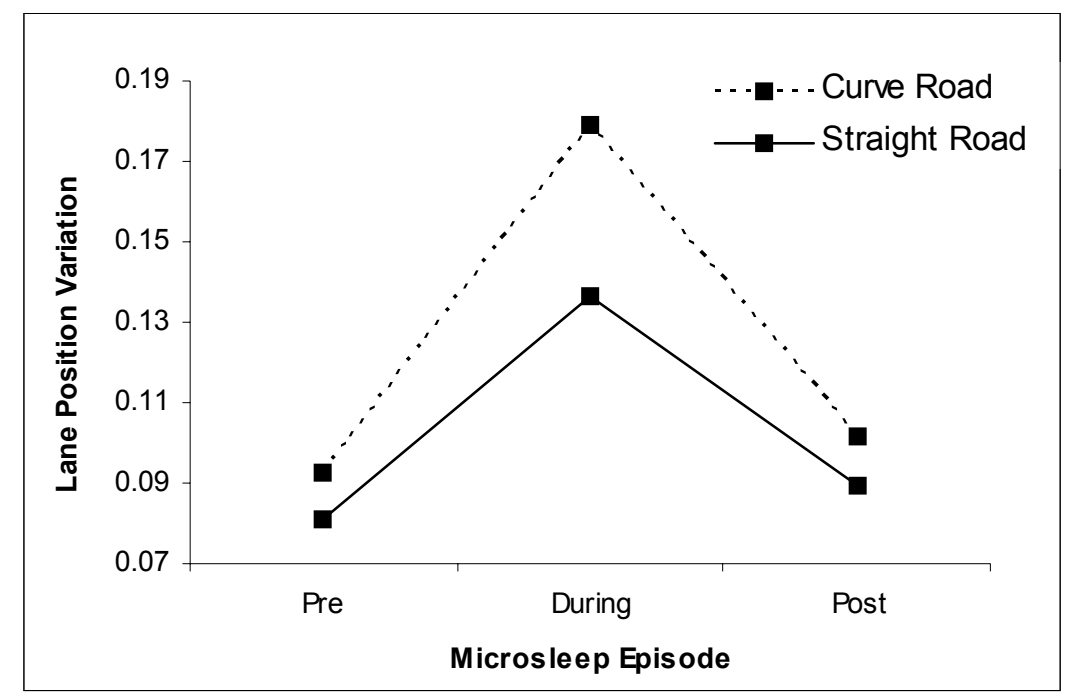

Figure 4. The effect of microsleeps on lane position variation for straight and curved roads

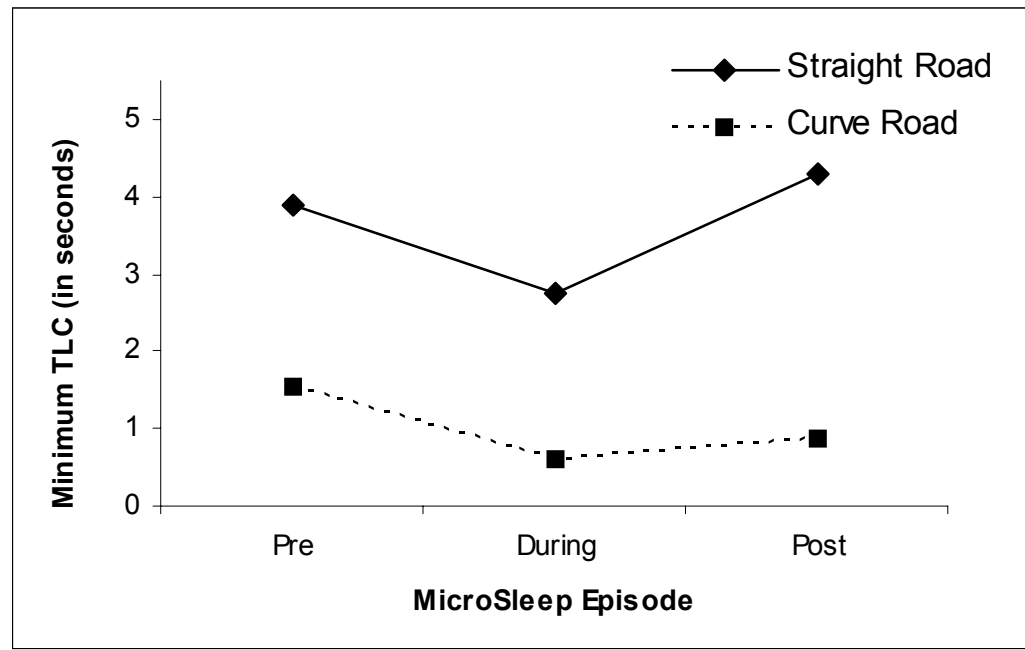

Figure 5. The effect microsleep on Minimum TLC for straight and curve roads 


\section{DISCUSSION}

The results of this study showed that driving performance measures (i.e., steering variation, and lane position variation, and minimum $T L C$ ) provide useful indicators of poorer driving control for OSAHS drivers with microsleep episodes. Drivers with OSAHS showed greater steering variation during microsleeps. Lower steering variation in the post-microsleep periods indicate that drowsy OSAHS drivers regain control after a microsleep ends. Reductions in steering control and low TLC in drowsy OSAHS drivers may lead to steering errors and lane breachment. Such errors can result in a crash with a vehicle in the opposite lane or an object on the side of the road. These risks are elevated during microsleep episodes on curves.

Increasing numbers of microsleeps have been associated with worsening overall simulated driving performance (Risser et al., 2000); however, direct effects of microsleeps on moment-tomoment vehicle control have not, to our knowledge, been reported. In this study, we established that EEG-defined microsleep episodes are associated with worse vehicle control in drowsy drivers with OSAHS. Microsleeps during uneventful segments do not always result in traffic conflicts, but increased exposure to microsleep episodes would be expected to increase a driver's crash risk, especially if the microsleeps occur on changing terrain (e.g., curves) or when other vehicles or hazards are present.

\section{REFERENCES}

American Thoracic Society. (1994). Sleep apnea, sleepiness, and driving risk. American Journal of Respiratory and Critical Care Medicine, 150, 1463-1473.

Findley, L., Unverzagt, M., Guchu, R., Fabrizio, M., Buckner, J., \& Suratt, P. (1995). Vigilance and automobile accidents in patients with sleep apnea or narcolepsy. Chest, 108(3), 619-624.

George, C. F. P., Nickerson, P. W., Hanly, P. J., Millar, T. W., \& Kryger, M. H. (1987). Sleep apnoea patients have more automobile accidents. The Lancet, 447.

Lin, C.-F., \& Ulsoy, A. G. (1995). Calculation of the time to lane crossing and analysis of its frequency distribution. Paper presented at Proceedings of the 1995 American Control Conference. Part 5 (of 6), Jun 21-23 1995, Seattle, WA, USA.

Liu, Schreiner, \& Dingus. (1999). Development of human factors guidelines for Advacnced Traveler Information Systems (ATIS) and Commercial Vehicle Operation (CVO): Human Factors Evaluation of the Effectiveness of Multimodality Displays in ATIS (No. FHWA-RD96-150): NHTSA.

Maclure, M. (1991). The Case-Crossover Design - a Method for Studying Transient Effects on the Risk of Acute Events. American Journal of Epidemiology, 133(2), 144-153.

Marshall, R. J. (1993). Analysis of case-crossover designs. Statistics in medicine, 12(24), 23332341.

Mollenhauer, M. A., Romano, R. A., \& Brumm, B. (2004, December, 2004). The Evaluation of a Motion Base Driving Simulator in a CAVE at TACOM. Paper presented at 24th Army Science Conference, Orlando, Florida. 
NHTSA. (2005). NHTSA's DROWSY DRIVER TECHNOLOGY PROGRAM. Retrieved April 23, 2005, from http://www-nrd.nhtsa.dot.gov/departments/nrd-01/summaries/ITS_11.html

Risser, M. R., Ware, J. C., \& Freeman, F. G. (2000). Driving simulation with EEG monitoring in normal and obstructive sleep apnea patients. Sleep, 23(3), 393-398.

Rizzo, M., McGehee, D., \& Jermeland, J. (2000). Design and installation of a driving simulator in a hospital environment. In K. Brookhuis, D. DeWaard \& C. Weikert (Eds.), Human System Interaction: Education, Research and Application in the 21st Century (pp. 69-77). Maastricht, The Netherlands: Shaker Publishing.

Young, T., Palta, M., Dempsey, J., Skatrud, J., Weber, S., \& Badr, S. (1993). The occurrence of sleep-disordered breathing among middle-aged adults. The New England Journal of Medicine, $328(17), 1230-1235$. 